\title{
A single phase modulation for pulse-based inductive- coupling connection in 3D stacked chip
}

\author{
Li Zhanga), Xiaowei Xu, Dawei Li, Xiaofei Chen, \\ and Xuecheng Zou \\ School of Optical and Electronic Information, \\ Huazhong University of Science and Technology, \\ Wuhan, China
}

a) andyzhang_ic@163.com

\begin{abstract}
This paper proposes an efficient modulation in high-speed interchip data communication with inductive-coupling wireless connection for 3D-stacked system in package ( $\mathrm{SiP})$. In this modulation, signal is generated only in one polarity of the digital signal transmission. Compared with BPM and NRZ modulation, it has a $50 \%$ power reduction and better crosstalk immunity.
\end{abstract}

Keywords: inductive-coupling, wireless connection, 3D package, modulation

Classification: Integrated circuits

\section{References}

[1] J. Burns, et al:: "Three-dimensional integrated circuits for low-power, highbandwidth systems on a chip," ISSCC Dig. Tech. Papers (2001) 268 (DOI: 10. 1109/ISSCC.2001.912632).

[2] T. Ezaki, et al.: "A $160 \mathrm{~Gb} / \mathrm{s}$ interface design configuration for multichip LSI," ISSCC Dig. Tech. Papers (2004) 140 (DOI: 10.1109/ISSCC.2004.1332633).

[3] R. J. Drost, et al.: "Proximity communication," CICC Proc. IEEE (2003) 469 (DOI: 10.1109/CICC.2003.1249442).

[4] N. Miura, et al.: “A $1 \mathrm{~Tb} / \mathrm{s} 3 \mathrm{~W}$ inductive-coupling transceiver for inter-chip clock and data link,” ISSCC Dig. Tech. Papers (2006) 1676 (DOI: 10.1109/ ISSCC.2006.1696223).

[5] N. Miura, et al.: “A $0.14 \mathrm{pJ} / \mathrm{b}$ inductive-coupling inter-chip data transceiver with digitally-controlled precise pulse shaping," ISSCC Dig. Tech. Papers (2007) 358 (DOI: 10.1109/ISSCC.2007.373442).

[6] K. Niitsu, et al.: “A $65 \mathrm{fJ} / \mathrm{b}$ inductive-coupling inter-chip transceiver using charge recycling technique for power-aware 3D system integration," ASSCC (2008) 97 (DOI: 10.1109/ASSCC.2008.4708738).

[7] N. Miura, et al.: “A $0.55 \mathrm{~V} 10 \mathrm{fJ} /$ bit inductive-coupling data link and $0.7 \mathrm{~V}$ $135 \mathrm{fJ} /$ Cycle clock link with dual-coil transmission scheme," IEEE J. SolidState Circuits 46 (2011) 965 (DOI: 10.1109/JSSC.2011.2108127).

[8] N. Miura, et al.: “A 195-Gb/s 1.2-W inductive inter-chip wireless superconnect with transmit power control scheme for 3-D-stacked system in a package," IEEE J. Solid-State Circuits 41 (2006) 23 (DOI: 10.1109/JSSC.2005.858625). 
[9] Y. Take, et al.: "A $30 \mathrm{~Gb} / \mathrm{s} /$ Link $2.2 \mathrm{~Tb} / \mathrm{s} / \mathrm{mm}$ inductively-coupled injectionlocking CDR for high-speed DRAM interface," IEEE J. Solid-State Circuits 46 (2011) 2552 (DOI: 10.1109/JSSC.2011.2164023).

[10] L. Zhang, et al.: "Analysis and measurement of misalignment effect in inductive-coupling wireless inter-chip connection," IEICE Electron. Express 14 (2017) 20170476 (DOI: 10.1587/elex.14.20170476).

\section{Introduction}

Inductive-coupling wireless connection is a promising interconnect technology for 3D stacked chips package. Compared with through silicon via (TSV) [1] connection, micro-bump connection [2] and capacitive-coupling connection [3], it has advantages such as: 1) this technology leads to low cost, high reliability and high yield without additional processes; 2) ESD protection is unnecessary, as its a non-contact interface; 3 ) the connection is current-driven, which means it can work under various supply voltages and processes; 4) There is no limitation in the number of stacked chips.

The power dissipation is a critical issue of inductive-coupling wireless connection. A lot of technologies has been developed to reduce the power consumption. Ref. [4] proposes a bi-phase modulation (BPM), gets $3 \mathrm{pJ} /$ bit power dissipation. Based on the BPM, some techniques have been developed for reducing the power furtherly. A Digitally-Controlled Precise Pulse Shaping technology is reported in ref. [5], got the power of $0.14 \mathrm{pJ} /$ bit. In ref. [6], a charge recycling technique is adopted, power is reduced to $65 \mathrm{fJ} / \mathrm{bit}$. In ref. [7], a dual-coil transmission scheme under low operating voltage reduced the power to $20 \mathrm{fJ} / \mathrm{bit}$. Ref. [8] proposed a non-return-to-zero (NRZ) modulation, gets $6 \mathrm{pJ} /$ bit power dissipation with 0.25 um CMOS process. There are two types of NRZ modulation, the synchronous type [8] and the asynchronous type [9]. The synchronous type NRZ modulation and BPM are pulse based modulation. They have a lower power dissipation comparing with non-pulse-based modulation, but their data rate is not high because of the requirement of precise timing control. This paper only discusses the pulse-based modulation.

This paper proposes a single-phase modulation (SPM), its transceiver scheme is presented in section 2. Performance analysis and comparison with BPM and NRZ modulation are in the section 3. Section 4 gives a conclusion.

\section{Single phase modulation}

Fig. 1 shows the schematic diagram and simulation waveform of the proposed SPM signaling transceiver. In the transmitter, a pulse generator produces pulse voltages whose pulsewidth is determined by the delay of the inverter chain. A pulse voltages is generated in every positive edge of $T_{X} c l k$. These pulse voltages do AND operation with $T_{X}$ data, pulse voltages can pass the AND gate only when the $T_{X}$ data is HIGH. Then the AND gate drives a NMOS transistor to convert the pulse voltages to pulse current $I_{T}$. When the pulse current flows through the $T_{X}$ inductor, 
induced voltage $V_{R}$ is produced in the $R_{X}$ inductor. The $R_{X}$ clk samples the $V_{R}$ in the first half of $V_{R}$, and recovers it to $R_{X} d a t a$.
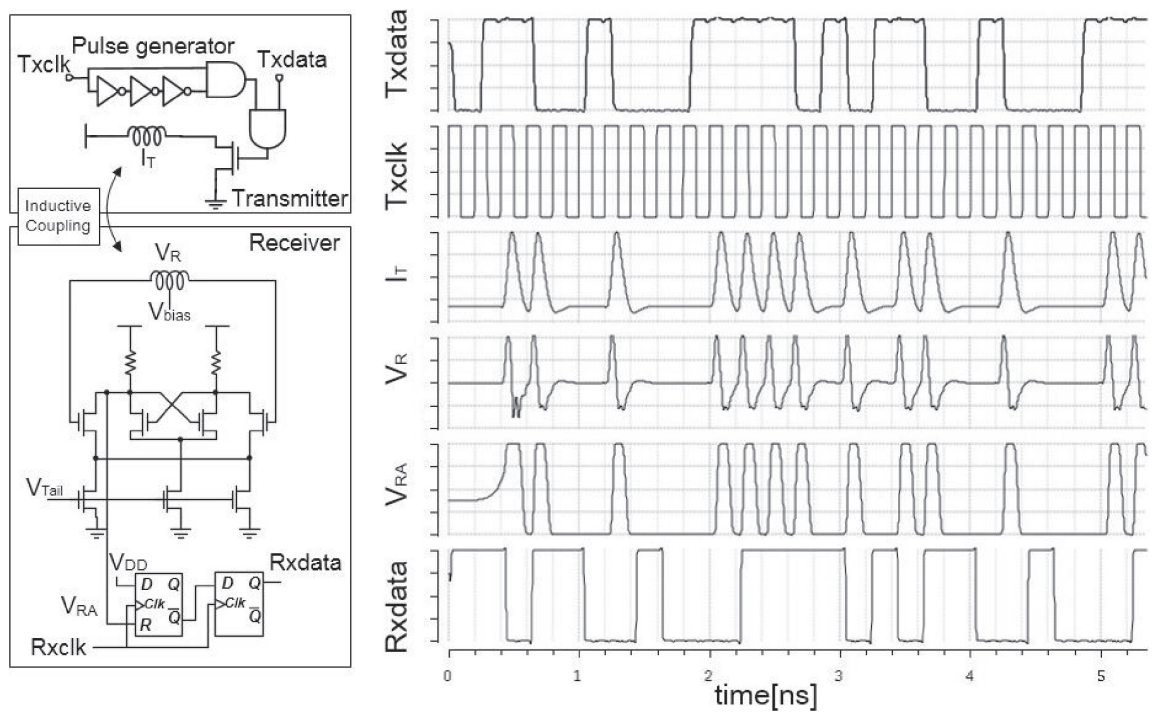

Fig. 1. Transceiver adopted SPM signaling and its simulation waveform

Fig. 2 shows the $I_{T}$ and $V_{R}$ waveform of NRZ, BPM and SPM signaling. In the BPM, every bit of data generates one current pulse. In the SPM, a pulse is produced only when the data " 1 " is transmitted. In the NRZ, the pulses are produced when the data changes. When the data turns from " 0 " to " 1 ", a positive pulse is produced. When the signal voltage turns to "0", a negative pulse is produced.

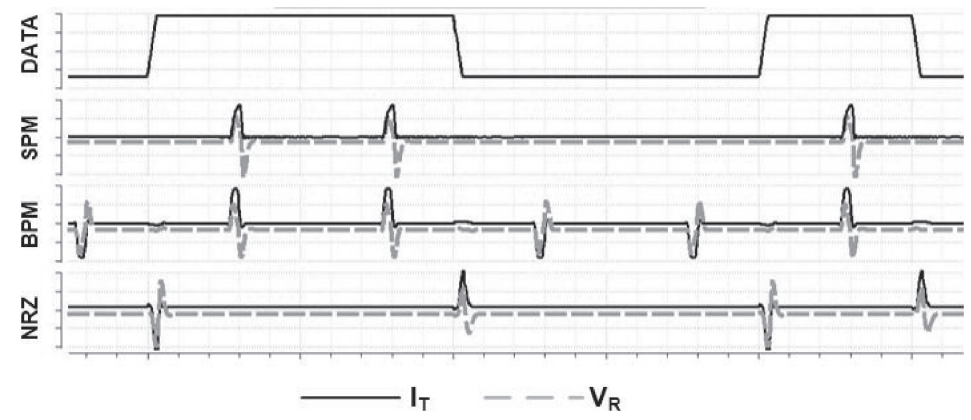

Fig. 2. Waveform comparison of SPM, BPM and NRZ

\section{Performance analysis and comparison}

\section{Power dissipation}

In the pulse based modulation for inductive coupling connection, energy is consumed by every current pulse. Fig. 3 shows the pulse shape of $I_{T}$ and the induced voltage $V_{R} . \tau$ is the width of $I_{T}, I_{P}$ is the peak current. $S_{P}$ is the slew rate of $I_{T}$. The energy consumed by the current pulse is approximately calculated by

$$
E_{\text {pulse }}=\frac{1}{2} V_{D D} \cdot I_{P} \cdot \tau=\frac{1}{2} V_{D D} \cdot S_{P} \cdot \tau^{2}
$$


The induced voltage is given by

$$
V_{P}=M \cdot \frac{d I_{T}}{d t} \approx M \cdot S_{P}=M \cdot \frac{I_{P}}{\tau}
$$

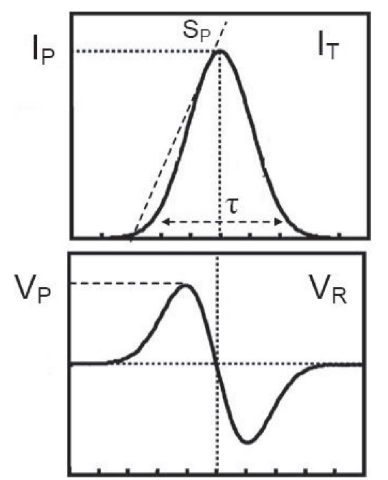

Fig. 3. Waveform of $I_{T}$ and $V_{R}$

$\mathrm{M}$ is the mutual inductance between two inductors. From eq. 1, 2, reducing the $\tau$ can not only lower the $E_{\text {pulse }}$, but also increase the $V_{R} . \tau$ is limited by requirement of bit error rate (BER). The too narrow pulsewidth will increase the probability of sampling errors. In the previous reports, $\tau$ should be larger than 120 ps. $E_{\text {pulse }}$ in ref. [4] is $0.11 \mathrm{pJ} /$ bit with $1 \mathrm{~Gb} / \mathrm{s}$ data rate.

In the BPM, energy consuming occurs in every bit of data. In the SPM, it only occurs when the data is "1". The power dissipation of SPM $P_{S P M}=P 1 * P_{B P M}, P 1$ is the proportion of " 1 " in the data. In a pseudo random binary sequence (PRBS), the quantity of " 1 " in the data is equal to that of "0", the power dissipation of SPM is $50 \%$ of BPM. In the NRZ modulation, the power dissipates when data changes. The power dissipation of NRZ is $P_{N R Z}=R R * P_{B P M}, R R$ is repeat rate of the data. In the PRBS, $R R=0.5$, the power dissipation of SPM is also $50 \%$ of BPM.

\section{Crosstalk immunity}

To obtain high bandwidth in a limited chip area, inductive-coupling connection channels are usually arranged in array with small spacing. The electromagnetic field of an inductor effects its adjoining inductors, mutual inductance are produced between inductors, which causes crosstalk. The received voltage with crosstalk is given by

$$
V_{R i}=Z_{\text {ind }}\left(k_{i i} I_{T}+\sum_{j \neq i} k_{i j} I_{T}\right)
$$

where $Z_{\text {ind }}$ is the trans-impedance of inductive coupling, $k_{i i}$ is the coupling coefficient between a $R_{X}$ inductor and its corresponding $T_{X}$ inductor. $k_{i j}$ is the coupling coefficient between the $R_{X}$ inductor and the non-corresponding $T_{X}$ inductor around it. Therefore, crosstalk-signal-ratio (CSR) is given by

$$
\operatorname{CSR}=\frac{k_{i i} I_{T}}{k_{i j} I_{T}}=\frac{k_{i i}}{k_{i j}}
$$


when the horizon distance between two inductors is larger than their diameter, the coupling coefficient between them is lower than zero. So $k_{i j}$ is a negative number. Which means that the crosstalk voltage produced by a positive current pulse is a negative pulse, as shown in Fig. 4.

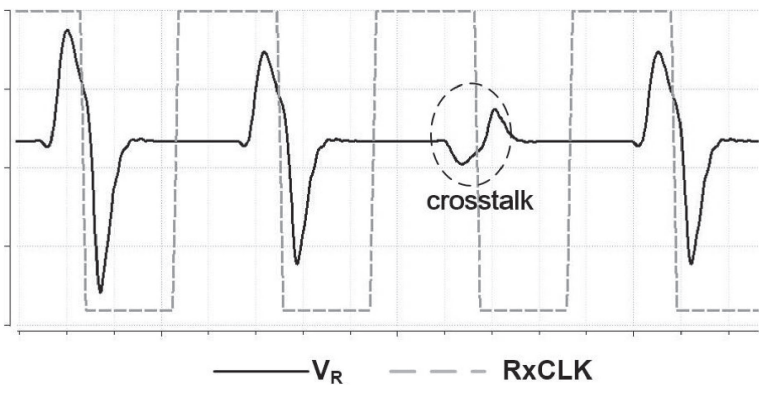

Fig. 4. Waveform of crosstalk

In BPM and NRZ modulation, there are two kinds of crosstalk pulse, the positive pulse and the negative pulse. In the worst condition, the crosstalk and noise always attenuate the signal, or produces a pulse in the place where there was no signal. The highest BER of NRZ, BPM are calculated by eq. 5, 6, respectively [4].

$$
\begin{aligned}
& B E R_{N R Z}=\frac{1}{2} \operatorname{erfc}\left(\frac{\tau}{4 \sqrt{2} \tau_{j i t t e r}} \sqrt{\ln \frac{1-N S R-C S R}{N S R+C S R}}\right) \\
& B E R_{B P M}=\frac{1}{2} \operatorname{erfc}\left(\frac{\tau}{4 \sqrt{2} \tau_{j i t t e r}} \sqrt{\ln \frac{1-N S R-C S R}{N S R}}\right)
\end{aligned}
$$

Where $\operatorname{erfc}()$ is the error function complement, $\tau$ is the $V_{R}$ pulsewidth, $\tau_{j i t t e r}$ is the jitter of sample clock, and NSR is the noise-to-signal ratio. NSR can be reduced by increasing transmit pulse energy. However, CSR remains constant because when the signal energy is increased, the crosstalk energy is increased simultaneously.

In SPM, there is only negative crosstalk pulse. That makes the condition of crosstalk in SPM more uncomplicated. In the signal "1", crosstalk attenuates the signal. In the signal " 0 ", the crosstalk produces a sag in the waveform (shown in Fig. 4), which makes the signal " 0 " safer. So the BER of SPM when signal "1" and " 0 " are calculated by eq. 7,8 , respectively.

$$
\begin{aligned}
& B E R_{S P M(1 \rightarrow 0)}=\frac{1}{2} \operatorname{erfc}\left(\frac{\tau}{4 \sqrt{2} \tau_{j i t t e r}} \sqrt{\ln \frac{1-N S R-C S R}{N S R}}\right) \\
& B E R_{S P M(0 \rightarrow 1)}=\frac{1}{2} \operatorname{erfc}\left(\frac{\tau}{4 \sqrt{2} \tau_{j i t t e r}} \sqrt{\ln \frac{1-N S R}{N S R}}\right)
\end{aligned}
$$

The highest BER of SPM, BPM and NRZ depending on transmit pulse energy are calculated with same parameters. The result is shown in Fig. 5, assuming the quantity of data "0" is equal to that of data "1". It can be seen that the BER of SPM is a slight lower than that of BPM, and the BER of NRZ is much higher than that of other two modulation. Because the uncertainty of the crosstalk in the NRZ makes the bit error happen easily, it needs some times higher power to get the same BER of SPM and BPM. 


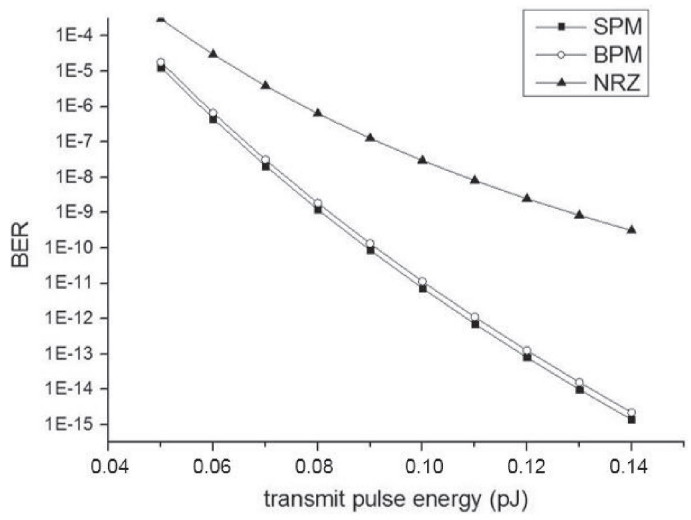

Fig. 5. Calculated BER of SPM, BPM and NRZ in the worst condition respectively $\left(C S R=0.2\right.$, Noise $=0.01 \mathrm{pJ}, \tau=125 \mathrm{ps}, \tau_{j i t t e r}=$ $25 \mathrm{ps})$

\section{Conclusions}

A single phase modulation for inductive-coupling connection has been introduced. This modulation reduces power dissipation significantly comparing with the BPM. Not be able to carry a clock is as a sacrifice. But when inductive-coupling connection channels are arranged by array, it's not necessary for each channel to carry a clock, and its advantage of good crosstalk immunity is shown up.

\section{Acknowledgments}

This work is supported by National Natural Science Foundation of China (Grant No. 61376031). 